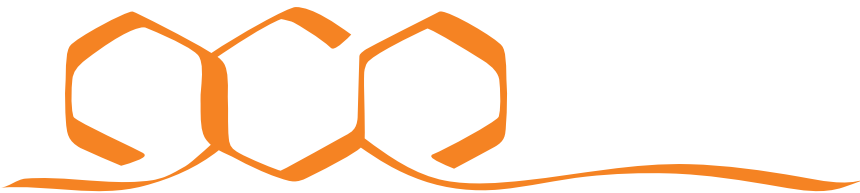 COMMUNICATIONS CHEMISTRY
}

ARTICLE

https://doi.org/10.1038/s42004-019-0202-8

\section{Liquid-liquid phase separation morphologies in ultra-white beetle scales and a synthetic equivalent}

\author{
Stephanie L. Burg (10 1, Adam Washington 1,2, David M. Coles ${ }^{1}$, Antonino Bianco ${ }^{3,4}$, Daragh McLoughlin ${ }^{5}$, \\ Oleksandr O. Mykhaylyk (1) 4, Julie Villanova ${ }^{6}$, Andrew J.C. Dennison', Christopher J. Hill ${ }^{7}$, Pete Vukusic ${ }^{8}$, \\ Scott Doak ${ }^{9}$, Simon J. Martin ${ }^{9}$, Mark Hutchings ${ }^{9}$, Steven R. Parnell ${ }^{10}$, Cvetelin Vasilev ${ }^{11}$, Nigel Clarke , \\ Anthony J. Ryan ${ }^{4}$, Will Furnass ${ }^{12}$, Mike Croucher ${ }^{13}$, Robert M. Dalgliesh (1) 2, Sylvain Prevost ${ }^{14,15}$, \\ Rajeev Dattani ${ }^{14}$, Andrew Parker ${ }^{16}$, Richard A.L. Jones ${ }^{1}$, J. Patrick A. Fairclough ${ }^{3}{ }^{3} \&$ Andrew J. Parnell $^{1}$
}

Cyphochilus beetle scales are amongst the brightest structural whites in nature, being highly opacifying whilst extremely thin. However, the formation mechanism for the voided intrascale structure is unknown. Here we report 3D x-ray nanotomography data for the voided chitin networks of intact white scales of Cyphochilus and Lepidiota stigma. Chitin-filling fractions are found to be $31 \pm 2 \%$ for Cyphochilus and $34 \pm 1 \%$ for Lepidiota stigma, indicating previous measurements overestimated their density. Optical simulations using finitedifference time domain for the chitin morphologies and simulated Cahn-Hilliard spinodal structures show excellent agreement. Reflectance curves spanning filling fraction of 5-95\% for simulated spinodal structures, pinpoint optimal whiteness for $25 \%$ chitin filling. We make a simulacrum from a polymer undergoing a strong solvent quench, resulting in highly reflective ( $94 \%)$ white films. In-situ X-ray scattering confirms the nanostructure is formed through spinodal decomposition phase separation. We conclude that the ultra-white beetle scale nanostructure is made via liquid-liquid phase separation.

\footnotetext{
${ }^{1}$ Department of Physics and Astronomy, The University of Sheffield, Sheffield S3 7RH, UK. ${ }^{2}$ ISIS Pulsed Neutron and Muon Source, Rutherford Appleton Laboratory, Harwell Science and Innovation Campus, Didcot OX11 OQX, UK. ${ }^{3}$ Department of Mechanical Engineering, The University of Sheffield, Sheffield S3 7HQ, UK. ${ }^{4}$ Department of Chemistry, Dainton Building, The University of Sheffield, Sheffield S3 7HF, UK. ${ }^{5}$ Akzo-Nobel, Decorative Paints Research and Development, Slough SL2 5DS, UK. ${ }^{6}$ ID16B Beamline, European Synchrotron Radiation Facility (ESRF), CS 40220, 38043 Grenoble Cedex 9 , France. ${ }^{7}$ Department of Biomedical Science, The University of Sheffield, Sheffield S10 2TN, UK. ${ }^{8}$ School of Physics, Exeter University, Exeter EX4 4QL, UK.

${ }^{9}$ Department of Materials, Loughborough University, Leicestershire LE11 3TU, UK. ${ }^{10}$ Faculty of Applied Sciences, Delft University of Technology, Mekelweg 15, 2629 JB Delft, The Netherlands. ${ }^{11}$ Department of Molecular Biology and Biotechnology, The University of Sheffield, Sheffield S10 2TN, UK. ${ }^{12}$ Department of Computer Science, The University of Sheffield, Sheffield S1 4DP, UK. ${ }^{13}$ Reserach Software Engineering Group, The University of Sheffield, Sheffield, UK. ${ }^{14}$ ID02 Beamline, European Synchrotron Radiation Facility (ESRF), F38043 Grenoble, France. ${ }^{15}$ Institut Laue-Langevin, 38042 Grenoble Cedex 9, France.

${ }^{16}$ Green Templeton College, University of Oxford, Woodstock Road, Oxford OX2 6HG, UK. Correspondence and requests for materials should be addressed to A.J.P. (email: a.j.parnell@sheffield.ac.uk)
} 
W hite surfaces are ubiquitous. Conventional white paint comprises a film-forming polymer latex dispersion loaded with high refractive index $(n \sim 2.6)$ nanoparticles of Titanium dioxide $\left(\mathrm{TiO}_{2}\right)$. In biology, however, whiteness is usually conferred by voided structures made of a continuous biopolymer phase and air. These biological "structural whites" 1,2 have been studied with a view to characterising their nanostructure and explaining their optical properties on the basis of their structure. The important question of how these structures arise in vivo, and the pathways by which they have evolved towards optimum optical properties, remains largely open ${ }^{3,4}$.

For the case of highly ordered crystalline photonic structures in beetle and butterfly scales, the structure formation has been postulated to be via pre-patterning and partitioning into intraand extra-cellular space, followed by chitin deposition ${ }^{5-8}$. Alternatively the patterning and deposition stages could possibly occur simultaneously ${ }^{9}$, although this process has not yet been seen in situ to fully understand the stages of photonic nanostructure development. The 3D photonic structures observed to date are ordered crystal grain structures that are highly faceted and strongly break up and segment the internal scale volume. These structures are proposed to be patterned using the physics of hydrophobic-hydrophilic interactions ${ }^{8}$ to control morphology (gyroid, cubic, hexagonal, sphere, lamellar etc.) akin to the structural diversity seen in photonic block copolymers ${ }^{10-12}$. These highly ordered 3D biological photonic structures exhibit multiple higher order X-ray scattering peaks due to their structural uniformity and order $^{8}$. However, previously published scattering data show that these higher order X-ray scattering peaks are not observed for the white beetles ${ }^{8}$.

Isotropic structural colour, that is independent of viewing angle, is widely found for pseudo-photonic crystals, which exhibit a degree of disorder. Here the optical properties are due to coherent light scattering from a two-phase (air and biopolymer) nanostructure. These are frequently observed in bird feathers ${ }^{13}$, and was first documented by Prum et al. ${ }^{14}$. The size of the welldefined length scale in these pseudo-photonic crystals directly affects the observed colour. This is clearly illustrated in Parnell et al. ${ }^{15}$, where Eurasian Jay feathers have a periodic gradient in colour from white to blue to black. Pseudo-photonic materials, which have a degree of disorder can be optically modelled using Bragg type reflecting structures, with a distribution of length scales in the layers and their spacings ${ }^{16}$. However, while such models may capture the optical properties well, they do not inform us as to the process by which these optically active nanomaterials form. It has been reported previously that blue structural colour in bird feathers and beetles is probably a result of biopolymer ${ }^{15,17,18}$ phase separation ${ }^{19}$ via spinodal decomposition with drying ${ }^{8}$ and chitin crystallisation ${ }^{20,21}$ being potential arrest mechanisms of phase separation ${ }^{19}$ within arthropod scales.

In this work, we show a complete ultra-structural characterisation of two intensely opaque biological systems-the white scales of the beetles ${ }^{22,23}$ Lepidiota stigma (L. stigma) and Cyphochilus. We compare these structures ${ }^{24}$ with those found in the late stage ${ }^{25}$ of the generic process of spinodal decomposition $^{26}$, and show by numerical solution of Maxwell's equation for these nanostructures that such spinodal structures can quantitatively reproduce the optical properties of the biological systems. This allows us to confirm that the beetle scales have close to the optimum combination of volume fraction and characteristic length scale to maximise their whiteness and fabricate a synthetic mimic using phase separation by spinodal decomposition.

\section{Results}

Non-destructive $\mathrm{X}$-ray nanotomography of ultra-white scales. The beetles, L. stigma and Cyphochilus, are covered with highly
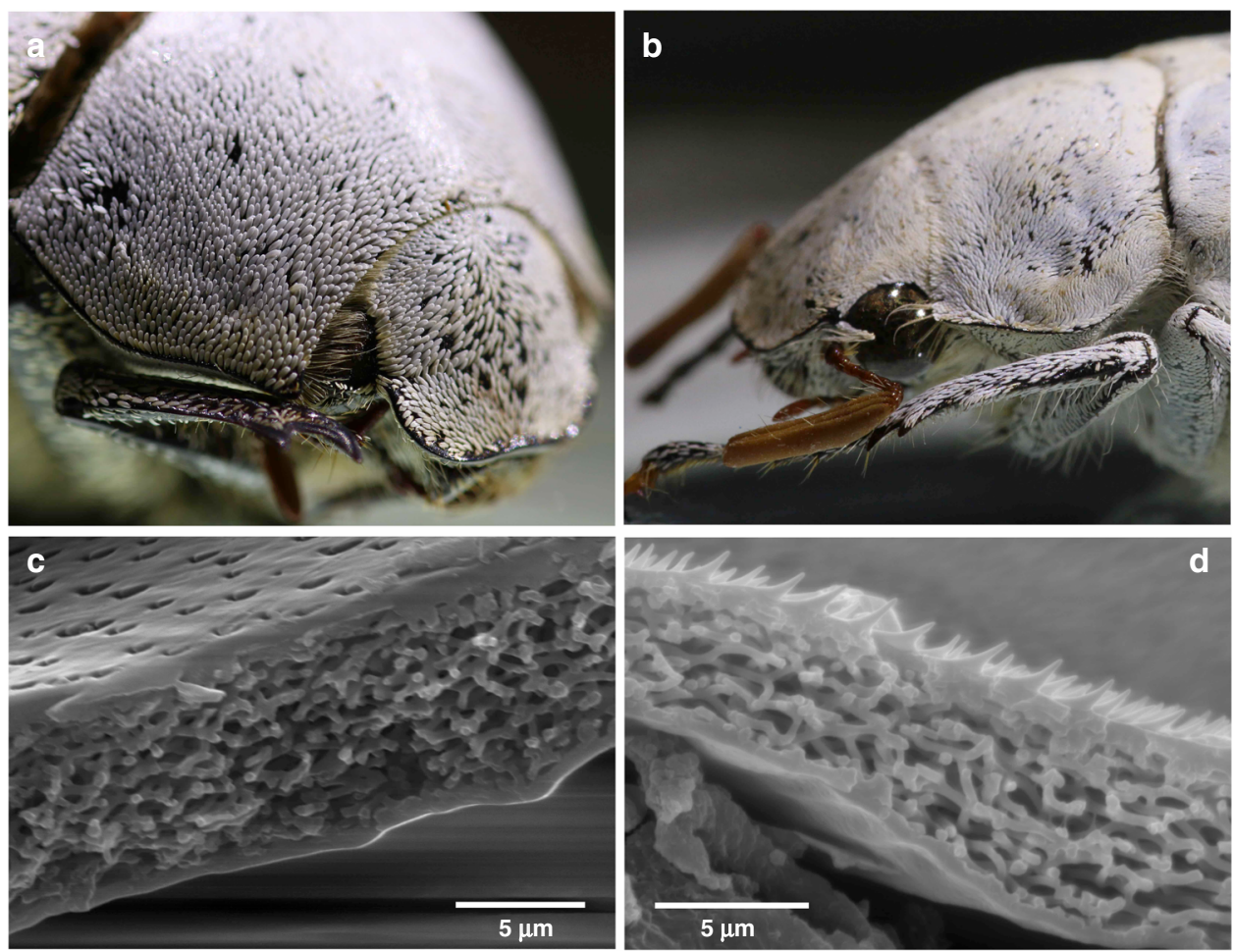

Fig. 1 Microscopy images of the ultra-white beetle scales. a An L. stigma beetle; the scales are $\sim 350 \mu \mathrm{m}$ long and $120 \mu \mathrm{m}$ wide. b An image of $a$ Cyphochilus beetle; the Cyphochilus scales are $\sim 220 \mu \mathrm{m}$ long and $60 \mu \mathrm{m}$ wide. The underlying black cuticle can be observed where there are no scales. c, $\mathbf{d}$ Crosssectional scanning electron microscopy (SEM) images of individual scales for L. stigma and Cyphochilus, respectively 

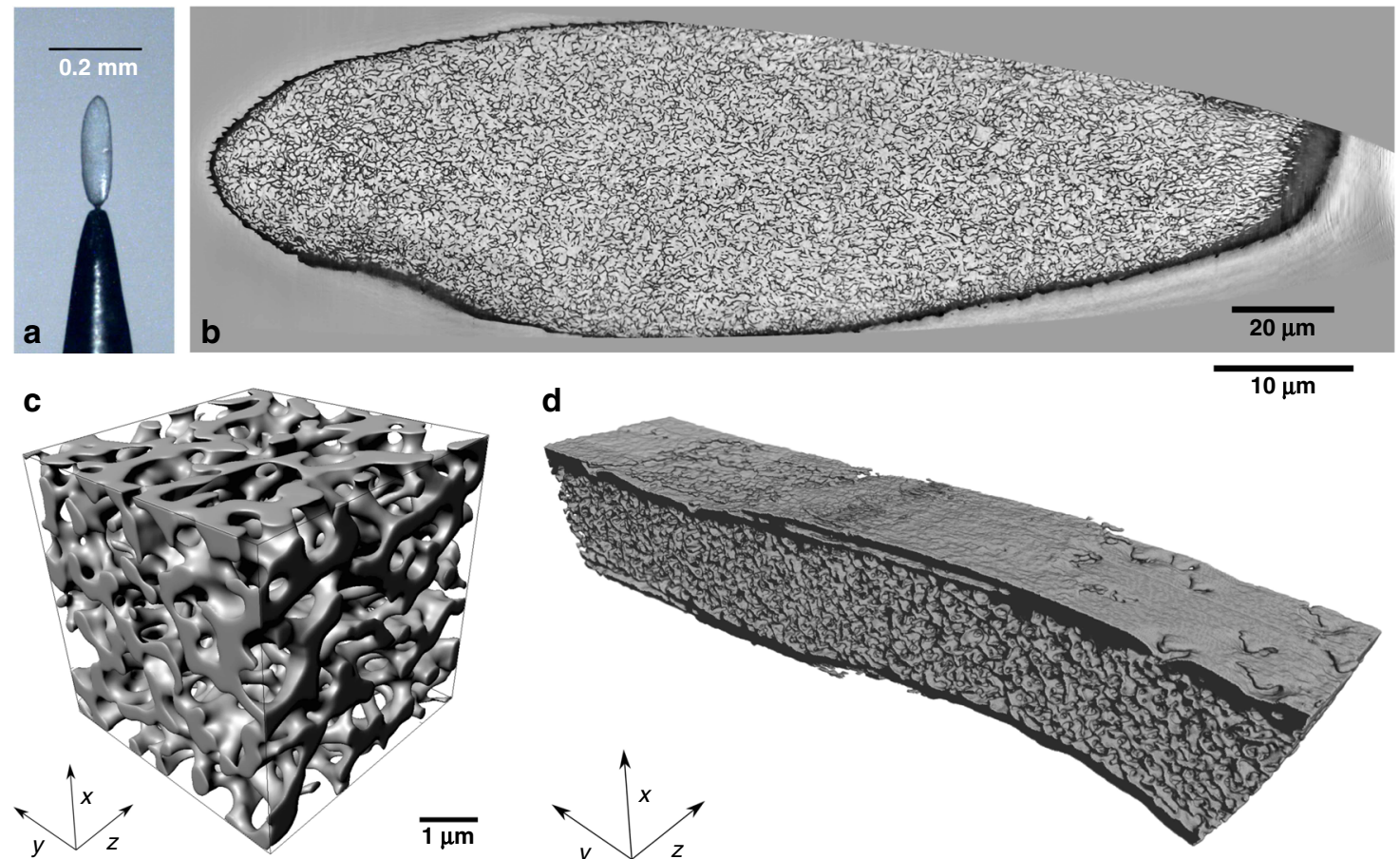

d

$10 \mu \mathrm{m}$
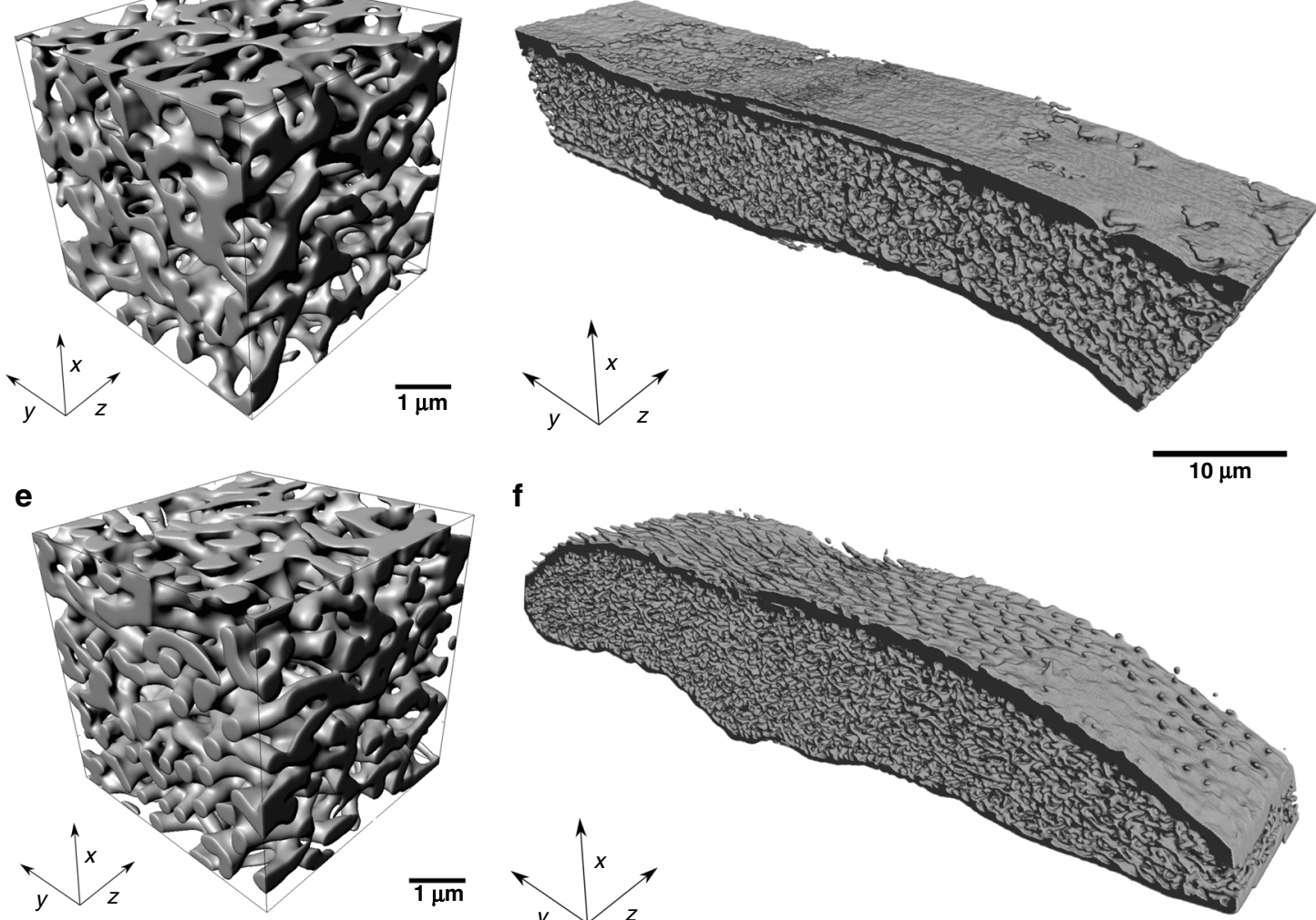

$\mathbf{f}$

$10 \mu \mathrm{m}$

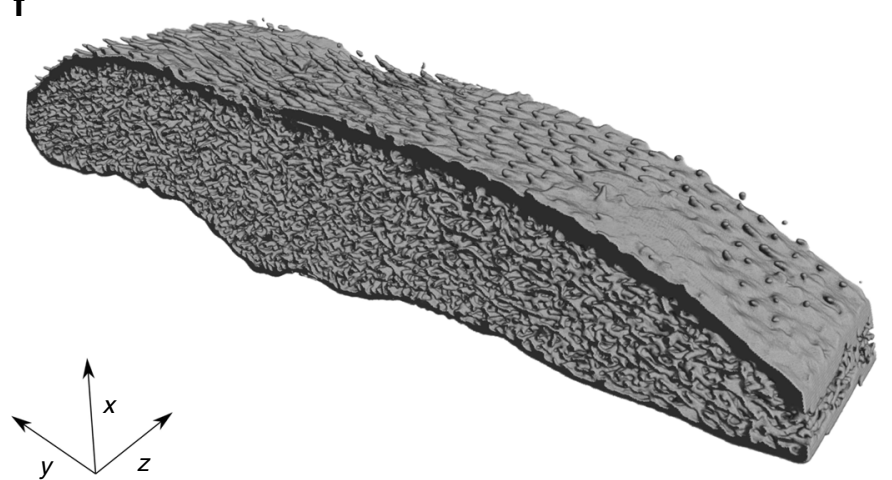

Fig. 2 X-ray nanotomography of intact ultra-white beetle scales. a Image showing a single intact Cyphochilus scale mounted to allow a full nanotomography scan. $\mathbf{b}$ Representative longitudinal slice ( $y-z$ plane) through the optical structure of an entire Cyphochilus scale. c Representative optical structure inside an L. stigma scale, based on the correlation analysis of 275 individual $5 \mu \mathrm{m}$ cubes. $\mathbf{d}$ A larger volume of $L$. stigma showing the extent and filling of the scale interior, and the surface features on the cuticle. e, $\mathbf{f}$ A typical cube and larger cross section, respectively, for the scale of $a$ Cyphochilus beetle

opaque, white scales (Fig. 1a, b). Their opacity arises from strong scattering throughout the internal structure of the scales. This inner scale space comprises an interconnected network of achitin (Supplementary Fig. 1) with a complementary interconnected network of air voids ${ }^{22}$. The internal structure of these white beetle scales was studied by scanning and transmission electron microscopy (SEM and TEM) revealing a "foam-like" structure of chitin fibrils (Fig. 1c, d), similar to that previously reported $^{22}$. We found that the sectioning of whole intact scales, even using focused ion beam (FIB) sectioning, resulted in severe scale distortion (Supplementary Figs. 2-4). The substantial warping and curving of the scales when they are cut open could be the result of the voided inner scale nanostructure having an inherent mechanical tension, which is relieved when the outer scale membrane becomes compromised. Therefore, in order to accurately model the optical properties of the scales, structural characterisation of intact scales is needed.

Imaging directly through the outer scale cuticle using X-ray nanotomography ${ }^{27}$ for individual Cyphochilus and L. stigma scales (Fig. 2) enabled the measurement of the full 3D internal morphology of complete intact scales (Fig. 2a). A cross section through the entire scale (Fig. 2b) shows that the internal structure is continuous, with no evidence of grain boundaries. This indicates that the process used to pattern the white beetles occurs at the same time point in time throughout the entire scale volume.

Representative $5 \mu \mathrm{m}$ cubes of the internal structure of the Cyphochilus and L. stigma are shown in Fig. 2c, e, respectively. These cubes were chosen from the 275 cubes cut from each full $3 \mathrm{D}$ dataset and had the minimum mean squared error compared with the average correlation functions shown later in Fig. 3a, b. Figure 2d, f shows scale cross sections; with thicknesses of $\sim 12 \mu \mathrm{m}$ for both species (Supplementary Figs. 5 and 6). The intrascale nanostructure is a highly continuous and interconnected air-voided network with a characteristic length scale, reminiscent of morphologies observed for phase separating polymer blends ${ }^{28}$, which have undergone phase separation via spinodal decomposition ${ }^{29-31}$. 
a

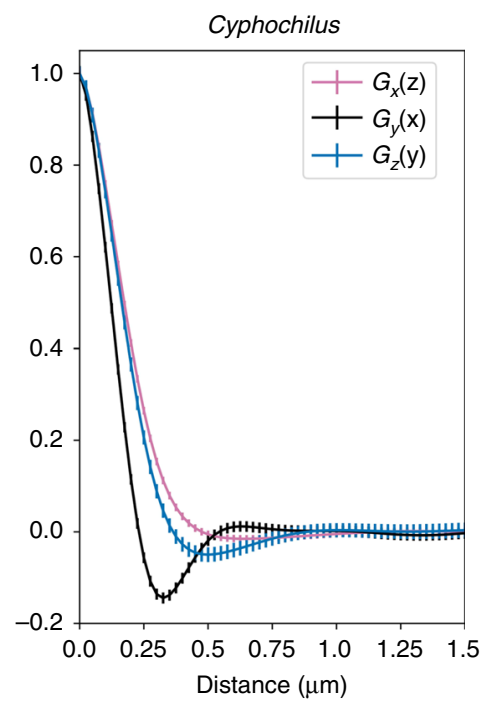

b

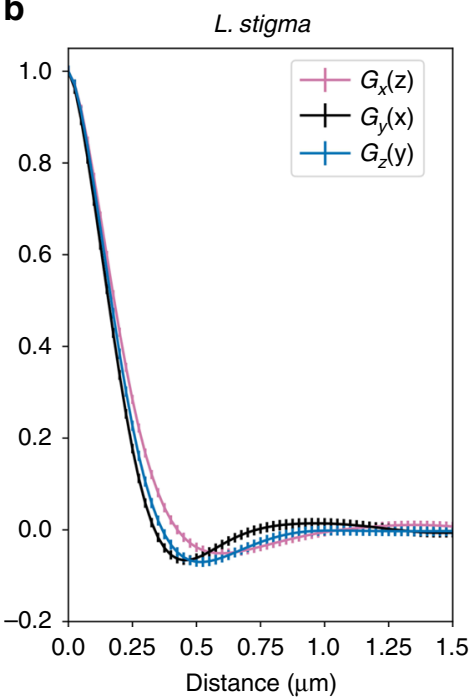

C

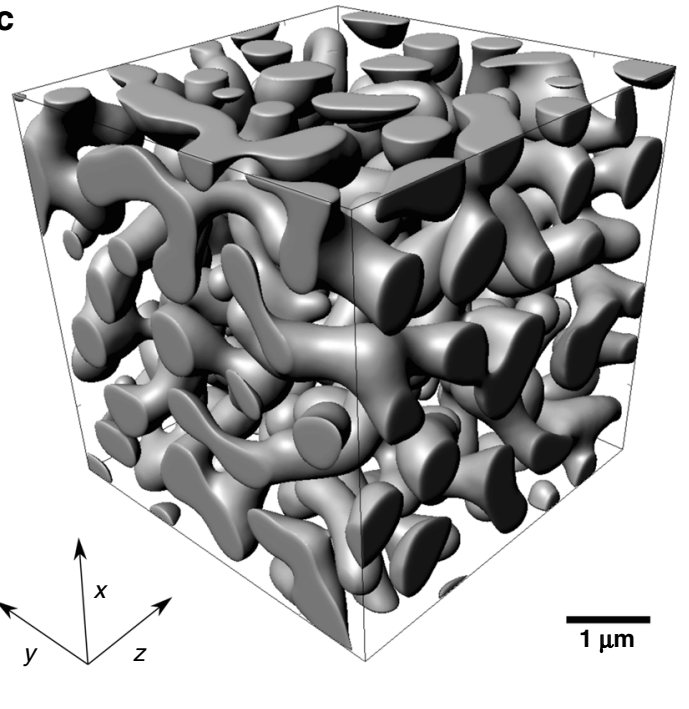

d

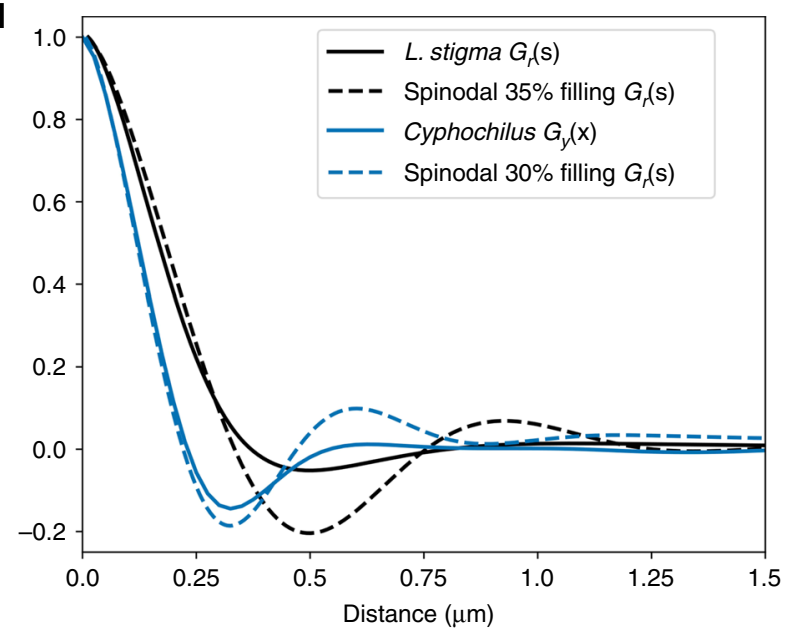

f

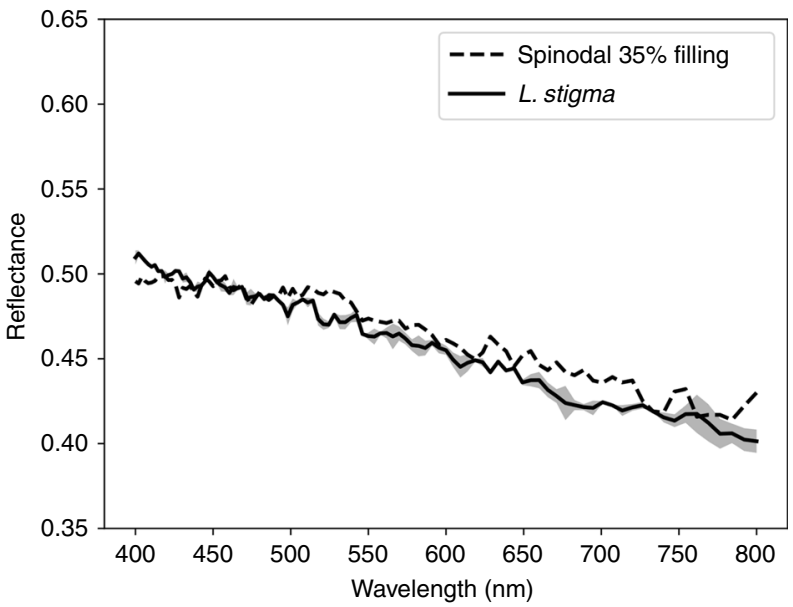

e

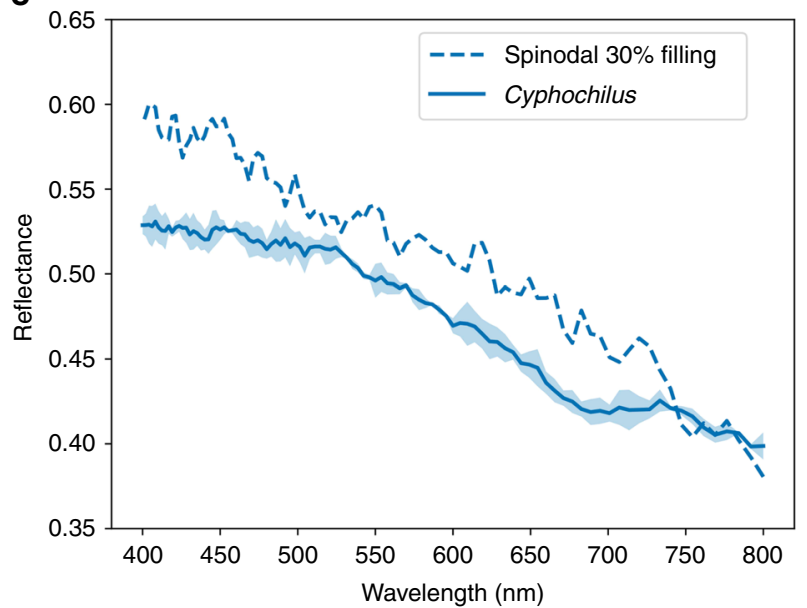

g

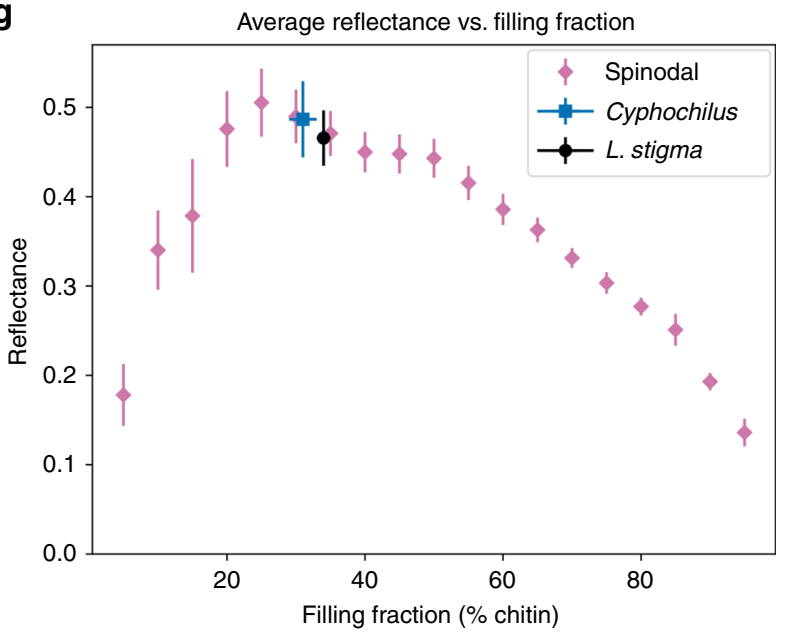

Fig. 3 Comparison of beetle scales and simulated spinodal whites. $\mathbf{a}, \mathbf{b}$ Projections of the correlation function in three orientations for the Cyphochilus and $L$. stigma intra-scale structures, respectively. Error bars represent the standard deviation at each point across the 275 averaged cubes. c A simulated latestage spinodal phase separated volume with a $30 \%$ volume-filling fraction, comparable to the Cyphochilus beetle scale-filling fraction. $\mathbf{d}$ Comparison of the correlation function projections for the measured beetle intra-scale morphologies and the (self-similar) spinodal simulations scaled to the same length scale. e Cyphochilus FDTD x-reflectance simulation for a slab of measured Cyphochilus intra-scale nanostructure and a simulated $30 \%$ filling-fraction spinodal with a $\mathrm{Gr}(\mathrm{s})$ scaled to $325 \mathrm{~nm}$ and $\mathbf{f}$ FDTD modelling showing theoretical $\mathrm{x}$-reflectance comparison for a 35\% filling-fraction spinodal scaled to $500 \mathrm{~nm}$ and the structure measured using X-ray nanotomography for L. stigma scales. $\mathbf{g}$ Average reflectance across the visible spectrum as a function of chitin-filling fraction from FDTD optical modelling. The confidence levels show the standard deviation of the reflectance across the sampled wavelengths $(400-800 \mathrm{~nm})$ for each filling fraction 
From the whole-scale nanotomography data we were able to determine the mean scale-filling fraction, based on entire scales, measured to be $31 \pm 2 \%$ for the Cyphochilus and $34 \pm 1 \%$ for the L. stigma. These values are lower than previously reported $4,23,32$, and lower than the values we measured using the FIB electron microscopy (Supplementary Tables 1 and 2). We attribute these differences to distortions arising from the use of sectioned scales in electron microscopy studies. Interestingly, our tomography results show only a very small difference of $3 \%$ in filling fraction between the two species, suggesting that there may be something special or limiting about a scale-filling fraction of $30 \%$, since the intra-scale structure of both species have converged towards this value.

Analysis of the X-ray nanotomography measured intra-scale structure provides insight into two important issues; the dominant length scale of the intra-scale structures, and the degree to which the structures are isotropic (Supplementary Note 1 and Supplementary Eqs. (1)-(9)). In the case of Cyphochilus, a degree of anisotropy is perceptible in Fig. 2e (and Supplementary Movie 1), where the $x-y$ plane contains significantly more small round fibril cross sections than the other two planes, indicating that fibrils preferentially lie in the $y-z$ plane (the plane parallel to the scale's largest surface). This is quantitatively shown in the average projections of the correlation function (Fig. 3a) along each axis for the 275 individual $5 \mu \mathrm{m}$ cubes for the Cyphochilus data. In the $x$-direction, shown by $\mathrm{G}_{\mathrm{y}}(\mathrm{x})$, the correlations are reduced when compared with the other two orientations. This is consistent with the literature ${ }^{33}$ and shows that the intra-scale structure for Cyphochilus is anisotropic, resulting in differences in the magnitude of the reflected wavelengths for the different axes (Supplementary Fig. 7), with light reflected strongest for the $x$-axis, but at the expense of the $y$ and $z$-axes. In the $x$-direction the correlation function has a much deeper minimum, indicating a prominent length scale in that direction. In addition, the location of the correlation minimum at $325 \mathrm{~nm}$ is somewhat smaller compared with the other two orientations, both of which occur at $500 \mathrm{~nm}$. In contrast to Cyphochilus, the correlation projections for L. stigma in Fig. 3b have similar correlation functions for each orientation, indicating that L. stigma scales have an isotropic internal structure (Supplementary Movie 2). This appears to be in contrast to previous studies ${ }^{23}$. Within error, the correlation minimum corresponds to a length scale of $500 \mathrm{~nm}$.

3D Cahn-Hilliard liquid-liquid phase separation simulations. To test if the optical properties of the ultra-white beetle scales could be replicated with a spinodal structure, we simulated a number of structures using numerical solutions in $3 \mathrm{D}$, of the Cahn-Hilliard equations for polymer systems in the long-time limit $^{25}$. We simulated effective chitin-filling fraction in the range between 5 and 95\%. These simulated structures capture the mechanism of length-scale selection present in spinodal decomposition, which leads to a structure that is random and isotropic, but with a characteristic length scale, together with the coarsening of that structure at late stages to a pattern obeying dynamic selfsimilarity $^{34}$ (Fig. 3c). In this regime, for a given filling fraction, the statistical characteristics of the patterns (pair correlation functions) are identical when scaled by a single characteristic length scale, which physically is determined by the length of time the system has had for domains to grow in size, known as coarsening 25 .

It was possible to set the characteristic length scale of the simulated late-stage spinodal structures to match the correlation functions of the measured beetle intra-scale structures (Fig. 3d). This was accomplished by adjusting the pixel to real-space conversion up and down in order to virtually zoom in and out of the structure. To match the Cyphochilus structure, a 30\% fillingfraction spinodal simulation was matched to the length scale in the $x$-axis Cyphochilus correlations. For the isotropic L. stigma structure, a $35 \%$ filling-fraction spinodal simulation was matched to the radially averaged L. stigma correlations.

Finite-difference time-domain optical modelling. To explore the optical properties of the reconstructed beetle intra-scale nanostructures, and the corresponding simulated spinodal structures, we needed to calculate reflection coefficients as a function of wavelength. This is a difficult regime in which to carry out such calculations-the presence of many strongly scattering interfaces separated by length scales comparable with optical wavelengths mean that many optical approximations are invalid, such as ray tracing ${ }^{35}$ or matrix methods ${ }^{36}$. Hence we used the full numerical solution of Maxwell's equations for the 3D structures using finite-difference time-domain methods (FDTD) ${ }^{37}$, this allowed us to calculate the broadband reflectance for any arbitrary structure.

To simulate the geometry of a scale lying on a beetle elytrum, $5 \mu \mathrm{m} \times 20 \mu \mathrm{m} \times 25 \mu \mathrm{m}(x, y, z)$ slabs of each beetle structure were cut from the tomography data, and FDTD was used to calculate the reflectance of the top of the slab in the $x$-direction. The same was done for slabs of the spinodal simulations with equivalent length scales and filling fractions to the intra-scale X-ray tomography data. The results for the $35 \%$ filling-fraction spinodal slab show excellent agreement with the reflectance results from the actual L. stigma structure (Fig. 3d). The Cyphochilus results were complicated by anisotropy in the intra-scale structure; however, the isotropic equivalent spinodal simulation was still able to capture the general trend of the Cyphochilus reflectance, by virtue of it exhibiting a higher reflectance for smaller incident wavelengths. It is important to stress that these results are not fits to the experimental data, but rather are FDTD simulations in which only the characteristic length scale and the filling fraction of the structure have been matched to the appropriate values for each beetle's measured intra-scale nanostructure, i.e. the reflectance of the beetles scales is predicted by a spinodal phase separation structure.

To investigate the effect of filling fraction for a fixed length scale, FDTD reflectance was calculated for slabs of Cahn-Hilliard spinodal structures with filling fractions covering the range of 5-95\%. The first minimum in correlations for all the slabs was set to $500 \mathrm{~nm}$, this being the length scale for the intra-scale structure of L. stigma measured via X-ray nanotomography. The results plotted as average scattering intensity over wavelengths $400-800 \mathrm{~nm}$ are presented in Fig. 3g. The reflectance from the measured beetle scale structures L. stigma (green point) and Cyphochilus (blue point) is also plotted to enable comparison, these both lie very close to their spinodal equivalents, the spinodal simulations reach maximum reflectance at $\sim 25 \%$ chitin-filling fraction. This important result, that filling fractions well below previously reported chitin scale-filling fractions result in superior reflectance, has very recently been reported for a naive simple branching random walk model ${ }^{38}$ despite being highly anisotropic. However, as the isotropic $30 \%$ filling-fraction spinodal simulation had a higher reflectance than the Cyphochilus structure (Supplementary Fig. 8). This shows that anisotropy is not crucial to achieving highly reflective materials, since the simulated isotropic spinodal structure is capable of superior reflectance. There has been an on-going debate ${ }^{39}$ in the field of opacifying layers as to whether "a flat" compressed nanofoam of air bubbles can outperform $\mathrm{TiO}_{2}$-based opacifying coatings. On the basis of these FDTD simulations we can see that this is not necessary and 

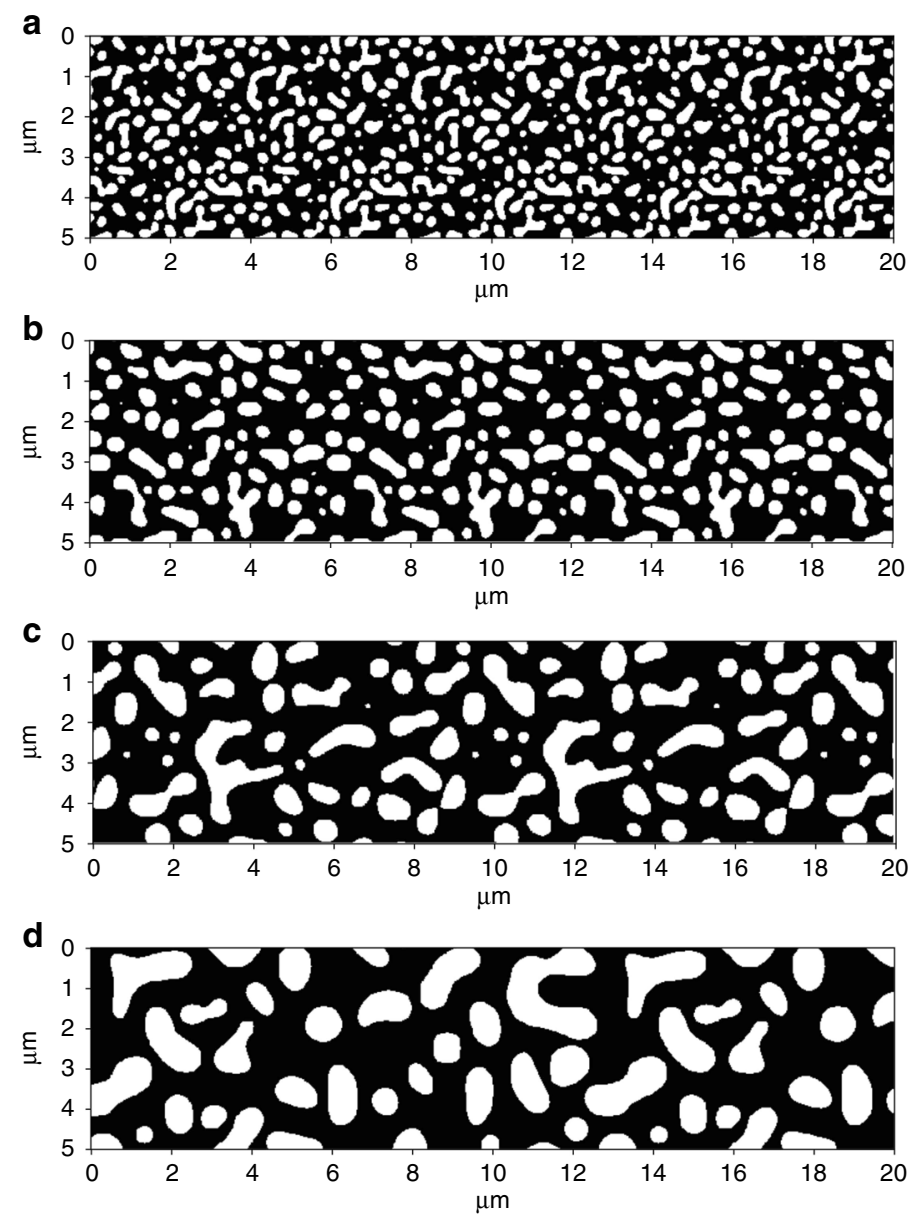
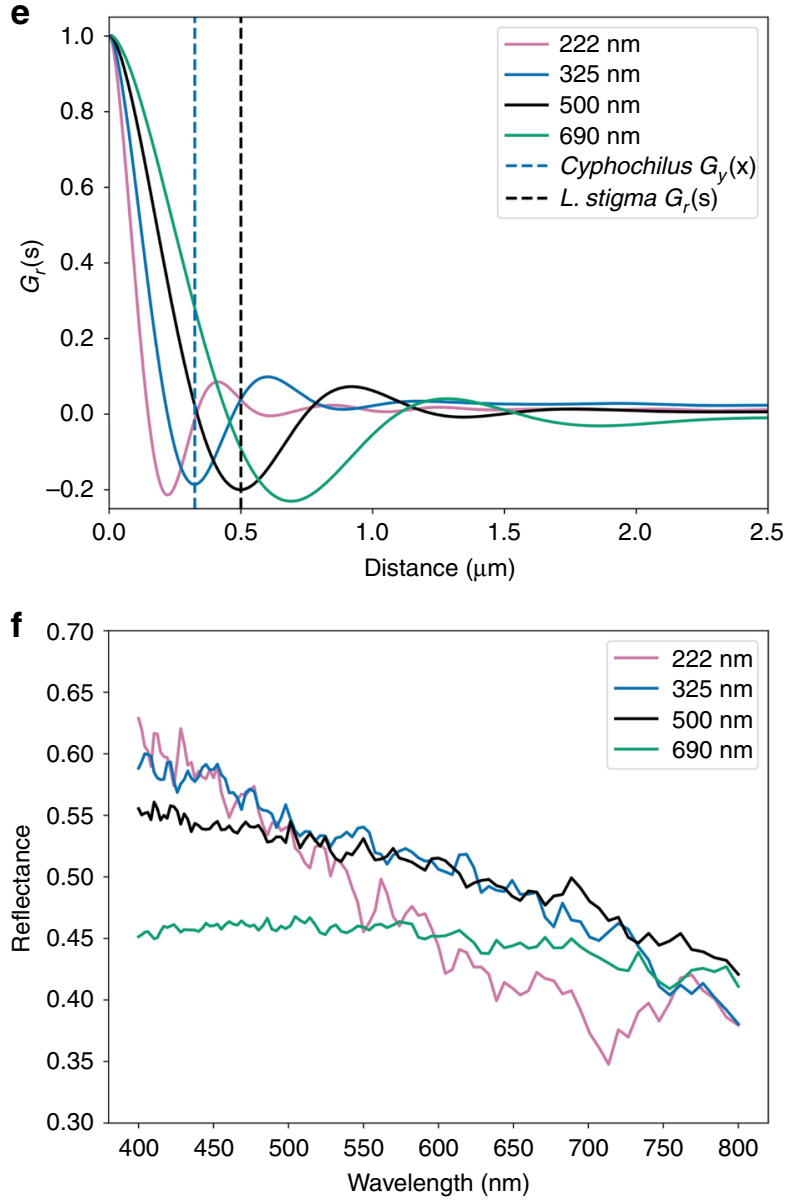

Fig. 4 Spectral response versus spinodal characteristic length scale. Representative 2D cross sections in the $x y$ plane for a series of spinodal slabs of varying characteristic length scale a $222 \mathrm{~nm}, \mathbf{b} 325 \mathrm{~nm}, \mathbf{c} 500 \mathrm{~nm}$ and $\mathbf{d} 690 \mathrm{~nm}$. Each slice, regardless of length scale is $5 \mu \mathrm{m} \times 20 \mu \mathrm{m}$ and has a filling fraction of $30 \%$ white pixels. e Gr(x) results for the 30:70 spinodal filling fraction for four different characteristic length scales. The dashed lines show the length scales of the Cyphochilus and the L. stigma beetles. f FDTD reflectance results for $5 \mu \mathrm{m} \times 20 \mu \mathrm{m} 30: 70$ spinodal optical structures having different characteristic correlation lengths

indeed the isotropic spinodal scale structure for both beetle length scales gives a higher level of reflectance, than the anisotropic equivalent.

The effect of length scale on the simulated reflectance for a fixed filling fraction is shown in Fig. 4. This was accomplished by altering the characteristic length scale of a $30 \%$ spinodal slab to four different values (Fig. $4 \mathrm{a}-\mathrm{d}$ ). The values were chosen such that two of the slabs matched the length scale of the Cyphochilus and L. stigma structures, one slab was smaller and one was larger (Fig. 4e). The FDTD reflectance results in Fig. 4f show a clear trend of increasing reflectance at low wavelengths with decreasing feature size at the cost of decreased reflectance at higher wavelengths. With its flat spectrum, the largest length scale $(690 \mathrm{~nm})$ would be the most white in colour, while the smallest length scale $(222 \mathrm{~nm})$ would be the most blue. If the aim of these structures is to maximise reflectance across all wavelengths (i.e. make the brightest white), and then a 500-nm length scale provides a suitable compromise between maximising reflectance, without losing reflected intensity at higher wavelengths and producing a more "blue-white" as observed for smaller length scales.

Based on the FDTD optical modelling we suggest that any synthetic route to create opacifying structures 40 via spinodal phase separation should aim for a filling fraction of $25 \%$ and a length scale of $500 \mathrm{~nm}$. While this optimal filling fraction is much lower than measured and reported for the beetle scales $4,23,32$ over the last 10 years, we propose that the high filling fraction values are an artefact of the assay, as the sample preparation causes densification as demonstrated by our comparative FIB SEM and $\mathrm{X}$-ray nanotomography results.

Nanostructured ultra-white cellulose acetate polymer film. Our deep structural understanding of the L. stigma and Cyphochilus intra-scale structures and their optical equivalence to structures formed by spinodal decomposition led us to develop a synthetic analogue. This was achieved using a commercially available bioderived polymer, cellulose acetate (CA). CA was dissolved in acetone, which underwent a solvent quench in the presence of a salt, calcium chloride $\left(\mathrm{CaCl}_{2}\right)$. The CA was molecularly dissolved in acetone to make a solution with a concentration of $0.1 \mathrm{~g} / \mathrm{mL}$ and mixed together with a methanol/salt solution such that the solvent ratio of acetone to methanol was $5: 3$ by volume. The solution was then drawn into a film and allowed to phase separate and vitrify, this process was fast $\sim 40 \mathrm{~s}$. The mass ratio of CA to salt was optimised to produce a film with the target filling fraction by control of the solvent quality. The optimum ratio was found to be $2: 1$ CA to salt by mass. 
a

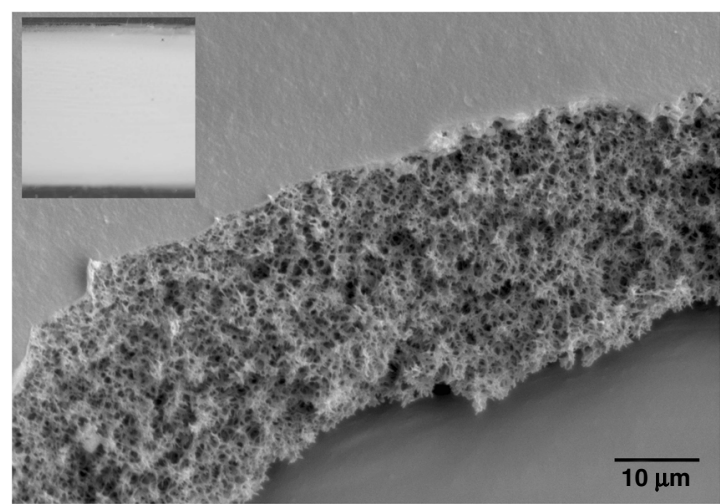

C

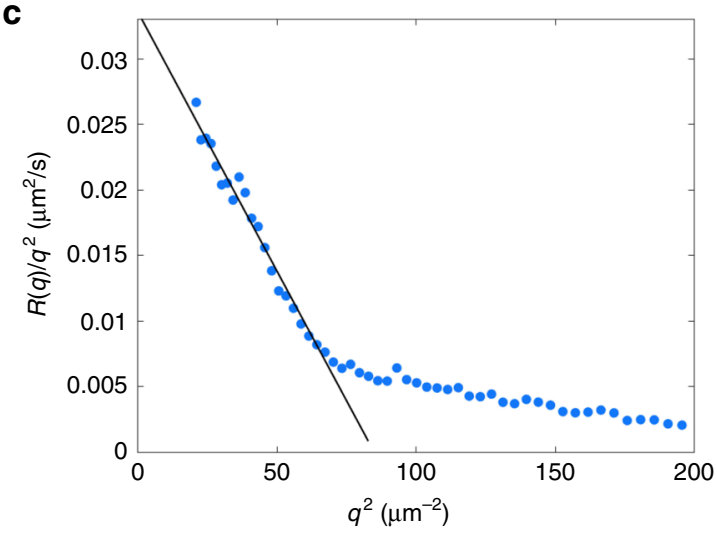

b

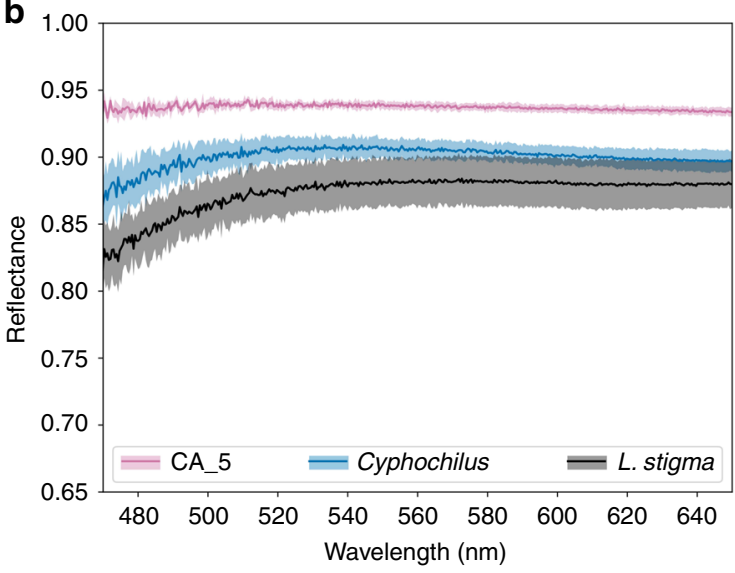

d

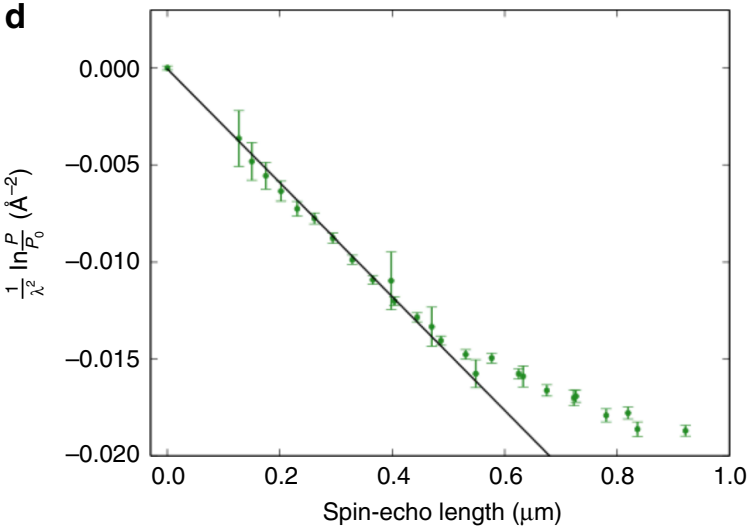

Fig. 5 Structure formation and characterisation of synthetic whites a SEM image of a porous white cellulose acetate film, with an inset showing the appearance of the white synthetic film. b Optical reflectance comparison for scales of L. stigma, Cyphochilus and an optimum cellulose acetate white film $(12.8 \pm 1.6 \mu \mathrm{m}$ in thickness) The shaded area represents the confidence levels calculated as the standard deviation across all measurements collected. $\mathbf{c}$ Amplification factor $R(q)$, measured by ultra small-angle X-ray scattering (USAXS) plotted as $R(q) / q^{2}$ versus $q^{2}$, showing a linear region typical of spinodal decomposition. $\mathbf{d}$ Linear fit to the initial slope of the wavelength normalised spin-echo small-angle neutron scattering (SESANS) $\mathrm{G}_{x}(\mathrm{y})$ signal, used to measure the density and therefore the filling fraction of the synthetic white films. The error bars are calculated based on the neutron counting statistics

The resulting CA films were white, highly voided (Fig. 5a), and exceedingly reflective as verified by reflectance measurements (Fig. 5b). Comparing the reflectivity of the CA film to scales from L. stigma and Cyphochilus for which reflectance is $90 \%$ and $88 \%$, respectively, the synthetic film had consistently higher reflectance, at $\sim 94 \%$ for a $12.8 \mu \mathrm{m}$ film (Supplementary Fig. 9), compared with both beetle species' scale thicknesses of $12 \mu \mathrm{m}$ at their thickest point.

To understand the structure formation mechanism we used time resolved, in situ, ultra small-angle X-ray scattering (USAXS). The fast data acquisition and unique USAXS length scale range at ID02 (European Synchrotron Radiation Facility (ESRF)) made it possible to follow the structural evolution and phase separation dynamics as a function of momentum transfer $q$, where $q=\frac{2 \pi}{d}, d$ being length scale. The early time information allowed us to measure the length-scale-dependent amplification factor $\mathrm{R}(\mathrm{q})$ at early times ${ }^{29,30}$ for our CA films. For longer length scales, a linear relationship between $\mathrm{R}(\mathrm{q}) / \mathrm{q}^{2}$ and $\mathrm{q}^{2}$ was found (Fig. 5c), confirming spinodal decomposition as the phase separation structuring mechanism.

Using spin-echo small-angle neutron scattering ${ }^{41,42}$ (SESANS) we were able to measure the correlation function directly for a large area of the synthetic structural white. This was performed in three orientations to confirm the isotropic nature of the CA films (Supplementary Fig. 10). The data showed a discontinuity
(Fig. 5d) at $\sim 470 \mathrm{~nm}$, close to the dominant length scale found for the ultra-white beetle internal scale structure. The density of the films could be determined from the slope of the correlation function and was found to be $21.5 \pm 4 \%$ (Fig. 5d, Supplementary Note 2 and Supplementary Eq. (10)).

Therefore, the improvement in the film's reflectance over the beetle scales is most likely the result of its filling fraction being close to the filling fraction required to achieve optimal reflectance, $25 \%$, determined using the filling fraction phase diagram (Fig. $3 \mathrm{~g}$ ). However, some of the increase in reflectance could be due to the specular component from the surface of the films and the slightly thicker voided layer, as the beetle scales possess a dense cuticle layer $\sim 1 \mu \mathrm{m}$ thick.

\section{Discussion}

In the case of the anisotropic network within the Cyphochilus, the internal a-chitin optical structure is highly interconnected and prone to distortion on sectioning, meaning that anisotropy must be imparted to the optical structure before it fully solidifies. We envision that the anisotropy is imparted in the late stage of liquid-liquid phase separation when the chitin precursors are transitioning to become mechanically stable crystalline $\alpha$-chitin, vitrifying the internal scale nanostructure. The anisotropy is probably caused by the liquid flow that removes the aqueous fluid from the narrow stalk opening back to the socket cell, leaving a 
dry desiccated $\alpha$-chitin air network. The effect of flow on phase separation is well known in spin-coated thin polymer blend films, where a radial flow provides a strong position dependent radial shear which alters the phase separation length scales and breaks the symmetry, due to advective flow ${ }^{43,44}$. There are several reasons this effect could be observed in the Cyphochilus and not the L. stigma such as differences in the liquid removal dynamics (shear flow) due to geometrical considerations (scale size) or the crystallisation of $\alpha$-chitin (reaction rate). In addition, the Cyphochilus nanostructure network has a small difference in filling fraction making it different mechanically and therefore possibly more susceptible to advective flow.

In summary, using FDTD optical modelling of the intact 3D intra-scale structures for L. stigma and Cyphochilus and simulated spinodal phase separated structures, we were able to establish design rules for producing highly reflective synthetic structural whites based on biological optical design. We then optimised the optical and structural properties of our cellulose acetate polymer films (controlling length-scale, filling fraction) and fabricated a synthetic mimic capable of producing a higher reflectance than the beetle scales for a comparable thickness. Given that we are able to replicate the morphology and optical reflectance of the beetle scales using structures that form via spinodal decomposition, using both optical simulations and the formation of a simulacrum, it seems nature uses the same liquid-liquid phase separation mechanism ${ }^{45}$ to create ultra-white structural colour.

The large existing body of work on structural colour has found a range of interesting and diverse structural colour architectures in biological systems. What has proved elusive has been the understanding of how these structures are made in vivo. Our insight into the optical structure of white beetle scales and our synthetic equivalent implicates liquid-liquid phase separation. This is increasingly seen as an important way to understand the process by which cell biology 45,46 provides order, organisation and segregation in cells across many different length scales. Our work confirms that some biological cells exhibiting structural colour use liquid-liquid phase separation to drive the formation of a highly interconnected bicontinuous nanonetwork in a single scale cell.

\section{Methods}

Fourier transform infrared attenuated total reflection. The beetle scales infrared (FTIR ATR) data were collected using a Thermo Scientific Nicolet iS10 Fourier transform infrared (FTIR) instrument equipped with a diamond attenuated total reflection (ATR). A background was collected; the sample was then placed in contact with the diamond ATR surface. A resolution of $2 \mathrm{~cm}^{-1}$ was used and a total of 128 acquisitions.

Scanning electron microscopy. SEM Beetle scales and the synthetic cellulose acetate samples were mounted using conductive carbon discs AGG3357N (Agar Ltd) on conductive metal stubs (Agar Ltd). These were sputtered with $\sim 10 \mathrm{~nm}$ of gold (Agar Ltd) and imaged on a JEOL JSM-6010LA using the InTouchScope data acquisition software.

Transmission electron microscopy. Scales from both the L. stigma and the Cyphochilus beetles were mounted in a 1:1 mixture of 1,2epoxypropane: Araldite resin and left overnight in a rotating mixer. The solvents were then replaced with fresh Araldite CY212 resin (Agar Scientific Ltd) and the samples were imbedded in 'coffin moulds'. The samples were allowed to cure at $60^{\circ} \mathrm{C}$ for $48-72 \mathrm{~h}$ before $500 \mathrm{~nm}$ sections were cut using an ultramicrotome (Reichert-Jung Ultracut E). The sections were subsequently stained with $1 \%$ toluidine blue in $1 \%$ borax. The sections were then washed with deionized water, dried and mounted using DPX (Agar Scientific). Finally, again using the ultramicrotome, sections were cut on 200 mesh copper grids and imaged using a TEM (FEI Tecnai). The thickness of the TEM sections was estimated at about $85-90 \mathrm{~nm}$. In order to determine the filling fraction of chitin within the scales the images needed were thresholded into binary images so the number of pixels corresponding to chitin in the images could be quantified.

Focused ion beam scanning electron microscopy. An FEI Nova 600 DualBeam FIB field emission gun scanning electron microscope from the Loughborough
Materials Characterisation Centre, was used for imaging. The gallium ion source ion in this instrument allowed regions to be milled away from the scale to reveal the internal network of chitin filaments. The probe current was kept between 3-7 pA. The Dual-Beam FIB was used to extensively study the internal structures and mill away and open the scales, slice layers from the samples (Supplementary Figs. 2-4).

X-ray nanotomography. The X-ray nanotomography on individual scales was performed at the ID16B beamline ESRF using holotomographic scheme with a high flux $\left(10^{12} \mathrm{ph} / \mathrm{s}\right)$ monochromatic beam $\left(\Delta \mathrm{E} / \mathrm{E}=10^{-2}\right)$ with an energy of $17.5 \mathrm{keV}$ The use of a $25 \mathrm{~nm}$ pixel size limits the field of view available to $54 \times 64 \times 64 \mu \mathrm{m}^{3}$. According to the sample geometry and dimensions, we mounted the samples in the vertical direction (Supplementary Figs. 11 and 12) in order to have the cross section fully in the horizontal field of view, and then performed multiple overlapping scans in the vertical direction. The Cyphochilus and the L. stigma scales were 250 and $350 \mu \mathrm{m}$, respectively, in length, which corresponded to six scans and eight scans in the vertical with $10 \mu \mathrm{m}$ overlap between the scans. For each acquisition, 2998 projections were acquired over $360^{\circ}$ with a counting time of $150 \mathrm{~ms}$ per projection. To avoid artefacts on the reconstructed images and keep the highest 3D resolution, the sample was mounted to give an unobstructed $360^{\circ}$ field of view. Beam damage was not observed. The reconstructed scans were then assembled and thresholded to separate the chitin and air pixels and produce the final 3D data sets (Supplementary Movies 3 and 4).

Finite-difference time-domain optical modelling. The FDTD simulations were performed using the commercial software, Lumerical (Lumerical Inc. Canada), which is a 2D- and 3D-solver for Maxwell's equations. For each simulation a 3D matrix containing a structure of interest represented as a binary matrix, where 1 was material and 0 was air. The material in every instance was taken to be chitin, which has a refractive index of 1.54 . Each matrix contained a slab of material that had a real-space size of $5 \mu \mathrm{m} \times 20 \mu \mathrm{m} \times 25 \mu \mathrm{m}$. To study how light propagated along each principle axis, the slabs were divided into 20 cubes, each $5 \mu \mathrm{m} \times 5 \mu \mathrm{m} \times$ $5 \mu \mathrm{m}$, and a polarised Gaussian source of width $1 \mu \mathrm{m}$ was directed into different faces of the cubes individually. Monitors collected the light, which exited the cubes, and the results were averaged across all of the cubes for a given slab. To study just the reflectance from a slab of material, an unpolarised Gaussian beam of width 6 $\mu \mathrm{m}$ was directed parallel to the shortest axis of the matrix and monitors collected the light, which was reflected back to the source. In all instances perfectly matched layers surrounded the region of interest, outside of the light monitors, to prevent light that had been scattered outside the region of interest from re-entering the simulation.

Synthetic films. To prepare the polymer solutions, cellulose acetate sheets (Clarifoil $^{\circ}$ ) were cut and weighted into a glass vial. Acetone (Sigma-Aldrich UK) was then added to the vial to achieve a target concentration of $0.1 \mathrm{~g} / \mathrm{mL}$. A stirrer flea was added and the vial was left to stir overnight at room temperature, to ensure the cellulose acetate was completely dissolved. In a separate vial, $\mathrm{CaCl}_{2}$ (SigmaAldrich) was dissolved in methanol (Sigma-Aldrich). The mass of $\mathrm{CaCl}_{2}$ was varied to the desired ratio by mass to cellulose acetate, the volume of methanol was set such that ratio between the acetone and methanol was $5: 3$ by volume. Finally, the $\mathrm{CaCl}_{2} /$ methanol solution was added to the cellulose acetate/acetone solution and left to stir at room temperature until homogeneous.

Once the solutions were prepared they were drawn into films of varying thicknesses. In order to accomplish this, an automated system was developed capable of achieving consistent and repeatable drawdowns. The system consisted of three main components, a drawdown cube, a drawdown guide to hold the cubes and substrate and a linear actuator to move the cubes across the substrate at constant speed.

Ultra small-angle $\mathbf{X}$-ray scattering. The USAXS measurements were performed at the ESRF, beamline ID02 ${ }^{47,48}$. X-rays with a wavelength of $1 \AA(12.4 \mathrm{keV})$ were used. The beam size was $20 \mu \mathrm{m} \times 20 \mu \mathrm{m}$, and sample-to-detector distance of 30.68 $\mathrm{m}$ ( $q$ range $0.002-0.2 \mathrm{~nm}^{-1}$ ). A set of rails was made to accommodate the drawdown cubes. The rails were on a moving platform that could rotate. The drawdown cube was positioned over the sample on the moving platform, maintaining the platform horizontal. As the cube finished a drawdown, the platform rotates into a vertical position, necessary for transmission USAXS (Supplementary Fig. 13).

Neutron reflectivity. Neutron Reflectivity (NR) was performed on a thin film of spin-coated cellulose acetate, spun from a $2 \mathrm{wt} \%$ acetonitrile (Sigma-Aldrich UK) solution. The substrate was a large diameter $(50.8 \mathrm{~mm})$ circular silicon wafer (Prolog Semicor, Ukraine). The NR data were measured at the ISIS pulsed Neutron and Muon Source (Oxfordshire, UK) using the instrument OFFSPEC with neutron wavelengths $2-12 \AA$. Three incident angles were collected $\left(0.5^{\circ}, 1.5^{\circ}\right.$ and $\left.2.5^{\circ}\right)$ to cover the momentum transfer range $0.008-0.238 \AA^{-1}$, using a resolution of $2.5 \%$. The data were reduced using Mantid ${ }^{49}$ and Offspec specific routines to stitch the three angles and subsequently rebin the data into one dataset. The data were then modelled using routines based on the scheme of Névot and Croce ${ }^{50}$ 
(Supplementary Fig. 14). From modelling a thin film of cellulose acetate has a measured scattering length density value of $1.64 \times 10^{-6} \AA^{-2}$

Spectroscopic ellipsometry. Spectroscopic ellipsometry (M2000V, J.A. Woollam) was used to determine the refractive index and absorption of a cellulose acetate thin film. A Cauchy ${ }^{51}$ model (Supplementary Eq. (11)) was used to fit $\Psi$, the ratio of the amplitude of the incident and reflected light beams and $\Delta$, the ratio of the phase lag of the incident and reflected light beams, over the wavelength range $375-1000 \mathrm{~nm}$. The model-derived optical constants are plotted in Supplementary Fig. 15, which shows that the absorption is negligible in the entire visible range.

Micro-spectroscopy measurements. The transmission/reflectance was measured using a bespoke microscope setup, we measured the light transmission for regions of individual beetle scales and the synthetic structured cellulose acetate films. The light source was a Tungsten light source, focused onto the sample using a long working lens $(100 \mathrm{~mm})$, the light was collected using a $\times 50$ objective (Mitutoyo Plan Apo SL infinity corrected) with a numerical aperture (NA) $=0.42$ and passed to an imaging spectrograph (Andor Shamrock SR193i). The data capture was performed using the Andor Solis software. A background without the lamp impinging on the spectrometer was collected to account for the electrical dark current. The optical transmission (T) through the glass substrate was collected for each sample measurement followed by the beetle scale/synthetic cellulose acetate region of interest. The field of view for each image entirely filled by a region of the beetle scale; a number of measurements were collected for Cyphochilus and $L$. stigma, as well as the cellulose acetate films. Reflectance was calculated as $1-\mathrm{T}$, which can be considered appropriate due to the negligible absorption of chitin ${ }^{52}$ and cellulose acetate (Supplementary Fig. 13) in the visible range and the excitation NA ( 0.08) being much smaller than collection NA (0.42).

Spin-echo small-angle neutron scattering. The beetle scale samples were prepared using a scalpel blade to remove elytra from Cyphochilus beetles. Individual elytra were stacked together and wrapped in Aluminium foil, which is transparent to thermal neutrons. The SESANS data for the Cyphochilus scales on the elytra were collected on the dedicated SESANS instrument at TU Delft in Holland. The number of wings required was determined through trial and error by increasing the number of wings in the beam until sufficient depolarisation of the beam was observed. The final number of elytra used was 28 . By estimating the number of scales inside a known area of the wing, an approximate scale density was calculated to be $\sim 9.7 \times 10^{-5}$ scales $/ \mu \mathrm{m}^{2}$, making a grand total of $\sim 3.7 \times 10^{5}$ scales for 28 elytra. The SESANS data for the CA films were taken on Larmor at the ISIS Spallation Neutron and Muon Source. A total of 30 films were sufficient for depolarisation of the beam. The films were measured in three different orientations with the films mounted vertically, horizontally and at an angle of $\sim 36^{\circ}$. The films were measured with neutrons with a wavelength range of $2-10 \AA$, a radio frequency of $1 \mathrm{MHz}$ and a beam size of about $5 \mathrm{~mm} \times 5 \mathrm{~mm}$. The data were collected with a count time of $30 \mathrm{~min}$ for magnetic field angles of 20,50 and $75^{\circ}$ giving a spin echo range out to $7 \mu \mathrm{m}$.

\section{Data availability}

All relevant data for this manuscript are available from the authors.

Received: 25 March 2019 Accepted: 2 August 2019

Published online: 29 August 2019

\section{References}

1. Mason, C. W. Structural colors in insects. I. J. Phys. Chem. 30, 383-395 (1926).

2. Dyck, J. Winter plumage of the rock ptarmigan: structure of the air-filled barbules and function of the white colour. Dan. Ornitolog. Foren. Tidsskr. 73, 41-58 (1979).

3. Zou, W. et al. Biomimetic polymer film with brilliant brightness using a onestep water vapor-induced phase separation method. Adv. Funct. Mater. 315, 1808885-1808888 (2019).

4. Wilts, B. D. et al. Evolutionary-optimized photonic network structure in White Beetle Wing Scales. Adv. Mater. 7, 1702057 (2017).

5. Ghiradella, H. Structure and development of iridescent butterfly scales: lattices and laminae. J. Morphol. 202, 69-88 (1989).

6. Ghiradella, H. Light and color on the wing-structural colors in butterflies and moths. Appl. Opt. 30, 3492-3500 (1991).

7. Saranathan, V. et al. Structure, function, and self-assembly of single network gyroid (I4132) photonic crystals in butterfly wing scales. Proc. Natl Acad. Sci. USA 107, 11676-11681 (2010)

8. Saranathan, V. et al. Structural diversity of arthropod biophotonic nanostructures spans amphiphilic phase-space. Nano Lett. 15, 3735-3742 (2015).
9. Wilts, B. D. et al. Butterfly gyroid nanostructures as a time-frozen glimpse of intracellular membrane development. Sci. Adv. 3, e1603119 (2017)

10. Edrington, A. C. et al. Polymer-based photonic crystals. Adv. Mat. 13, 421-425 (2001).

11. Parnell, A. J. et al. Shear ordered diblock copolymers with tuneable optical properties. Phys. Chem. Chem. Phys. 13, 3179-3186 (2011).

12. Parnell, A. J. et al. Continuously tuneable optical filters from self-assembled block copolymer blends. Soft Matter 7, 3721 (2011).

13. Saranathan, V. et al. Structure and optical function of amorphous photonic nanostructures from avian feather barbs: a comparative small angle X-ray scattering (SAXS) analysis of 230 bird species. J. R. Soc. Interface 9, 2563-2580 (2012).

14. Prum, R. O., Torres, R. H., Williamson, S. \& Dyck, J. Coherent light scattering by blue feather barbs. Nature 396, 28-29 (1998).

15. Parnell, A. J. et al. Spatially modulated structural colour in bird feathers. Sci. Rep. 5, 18317 (2015).

16. Meiers, D. T., Heep, M. C. \& Freymann, V. G. Invited article: Bragg stacks with tailored disorder create brilliant whiteness. APL Photonics 3, 100802-100807 (2018).

17. Dufresne, E. R. et al. Self-assembly of amorphous biophotonic nanostructures by phase separation. Soft Matter 5, 1792-1795 (2009).

18. Dong, B. Q. et al. Optical response of a disordered bicontinuous macroporous structure in the longhorn beetle Sphingnotus mirabilis. Phys. Rev. E 84, 011915 (2011)

19. Alberti, S. Phase separation in biology. Curr. Biol. 27, R1097-R1102 (2017).

20. Bracker, C. E., Ruiz-Herrera, J. \& Bartnicki-Garcia, S. Structure and transformation of chitin synthetase particles (chitosomes) during microfibril synthesis in vitro. Proc. Natl Acad. Sci. USA 73, 4570-4574 (1976).

21. Merzendorfer, H. Insect chitin synthases: a review. J. Comp. Physiol. B 176, 1-15 (2005)

22. Vukusic, P., Hallam, B. \& Noyes, J. Brilliant whiteness in Ultrathin Beetle Scales. Science 315, 348-348 (2007).

23. Burresi, M. et al. Bright-white beetle scales optimise multiple scattering of light. Sci. Rep. 4, 6075 (2014).

24. Cahn, J. W. Phase separation by spinodal decomposition in isotropic systems. J. Chem. Phys. 42, 93-99 (1965).

25. Huse, D. Corrections to late-stage behavior in spinodal decomposition: Lifshitz-Slyozov scaling and Monte Carlo simulations. Phys. Rev. B Condens. Matter 34, 7845-7850 (1986)

26. Bray, A. J. Theory of phase-ordering kinetics. Adv. Phys. 51, 481-587 (2010)

27. Martinez-Criado, G. et al. ID16B: a hard X-ray nanoprobe beamline at the ESRF for nano-analysis. J. Synchrotron Radiat. 23, 344-352 (2016).

28. Ebbens, S. et al. In situ imaging and height reconstruction of phase separation processes in polymer blends during spin coating. ACS Nano 5, 5124-5131 (2011).

29. Cook, H. E. Brownian motion in spinodal decomposition. Acta Metall. 18 297-306 (1970)

30. Bates, F. S. \& Wiltzius, P. Spinodal decomposition of a symmetric critical mixture of deuterated and protonated polymer. J. Chem. Phys. 91, 3258-3274 (1989).

31. Burg, S. L. \& Parnell, A. J. Self-assembling structural colour in nature. J. Phys Condens. Matter 30, 413001-413021 (2018).

32. Luke, S. M., Hallam, B. T. \& Vukusic, P. Structural optimization for broadband scattering in several ultra-thin white beetle scales. Appl. Opt. 49, 4246-4254 (2010).

33. Cortese, L. et al. Anisotropic light transport in White Beetle Scales. Adv. Opt Mater. 3, 1337-1341 (2015).

34. Hashimoto, T., Itakura, M. \& Hasegawa, H. Late stage spinodal decomposition of a binary polymer mixture. I. Critical test of dynamical scaling on scattering function. J. Chem. Phys. 85, 6118-6128 (1986).

35. Vallone, M. et al. Comparing FDTD and ray-tracing models in numerical simulation of HgCdTe LWIR photodetectors. J. Electron. Mater. 45 4524-4531 (2016)

36. Krumpholz, M., Huber, C. \& Russer, P. A field-theoretical comparison of Fdtd and Tlm. IEEE Trans. Microw. Theory Tech. 43, 1935-1950 (1995).

37. Yee, K. Numerical solution of initial value problems of Maxwells equations. IEEE Trans. Antennas Propag. 14, 302-307 (1966)

38. Utel, F., Cortese, L., Wiersma, D. S. \& Pattelli, L. Optimized white reflectance in photonic network structures. Adv. Opt. Mater. 7, 1900043 (2019).

39. Ross, W. D. Theoretical computation of light scattering power: comparison between TiO2 and air bubbles. J. Paint Technol. 43, 49-66 (1971).

40. Syurik, J., Jacucci, G., Onelli, O. D., Hölscher, H. \& Vignolini, S. Bio-inspired highly scattering networks via polymer phase separation. Adv. Funct. Mater. 28, 1706901-1706906 (2018).

41. Andersson, R., Heijkamp, L. F. V., Schepper, I. M. \& de, Bouwman, W. G. IUCr. Analysis of spin-echo small-angle neutron scattering measurements. $J$. Appl. Cryst. 41, 868-885 (2008). 
42. Parnell, S. R. et al. Porosity of silica Stöber particles determined by spin-echo small angle neutron scattering. Soft Matter 12, 4709-4714 (2016).

43. Travasso, R. D. M., Buxton, G. A., Kuksenok, O., Good, K. \& Balazs, A. C. Modeling the morphology and mechanical properties of sheared ternary mixtures. J. Chem. Phys. 122, 194906-194912 (2005).

44. Liu, J., Dedè, L., Evans, J. A., Borden, M. J. \& Hughes, T. J. R. Isogeometric analysis of the advective Cahn-Hilliard equation: spinodal decomposition under shear flow. J. Comput. Phys. 242, 321-350 (2013).

45. Alberti, S., Gladfelter, A. \& Mittag, T. Considerations and challenges in studying liquid-liquid phase separation and biomolecular condensates. Cell 176, 419-434 (2019).

46. Shin, Y. \& Brangwynne, C. P. Liquid phase condensation in cell physiology and disease. Science 357, 1-11 (2017).

47. Van Vaerenbergh, P. et al. An upgrade beamline for combined wide, small and ultra small-angle X-ray scattering at the ESRF. AIP Conf. Proc. 1741, 030034-030035 (2017)

48. Narayanan, T. et al. A multipurpose instrument for time-resolved ultra-smallangle and coherent X-ray scattering. J. Appl. Cryst. 51, 1511-1524 (2018)

49. Arnold, O. et al. Mantid-Data analysis and visualization package for neutron scattering and $\mu$ SR experiments. Nucl. Inst. Methods Phys. Res. A 764, 156-166 (2014).

50. Nevot, L. \& Croce, P. Caractérisation des surfaces par réflexion rasante de rayons X. Application à l'étude du polissage de quelques verres silicates. Rev. Phys. Appl. 15, 761-779 (1980).

51. Gooch, J. W. (ed) Encyclopedic Dictionary of Polymers 167-167 (Springer, New York, 2007).

52. Leertouwer, H. L., Wilts, B. D. \& Stavenga, D. G. Refractive index and dispersion of butterfly chitin and bird keratin measured by polarizing interference microscopy. Opt. Express 19, 24061-24066 (2011).

\section{Acknowledgements}

This project was co-funded by Innovate UK (Grant Number 33692-239251) in partnership with AkzoNobel and the University of Sheffield. We are grateful to Dr Andrew Burgess, Dr Gareth Crapper, Dr David Elliott and Dr Phil Taylor all from AkzoNobel for initiation of the project and their continued support throughout this work. We also acknowledge the Science and Technologies Facilities Council for access to facilities at the ESRF via experiments SC 4363 and SC 4386 and at the ISIS Spallation Neutron Source under experiment RB 810719 (data available at https://doi.org/10.5286/ISIS.E.92924017) and RB 1769002 (NWO Dutch Access) via TU Delft.

\section{Author contributions}

A.J.P., D.M., R.A.L.J. and J.P.A.F. conceived the study. A.P. selected from a cross section of opacifying structures from the NHM. A.J.P performed SEM of Beetle scales, optical microscopy and reflectance measurements with C.V. FIBSEM work and analysis was carried out by S.J.M., S.D. and M.H. TEM of the Beetle scales by C.H. S.B., A.W., A.D and J.V. performed the X-ray tomography at the ESRF. S.B. reconstructed the data, and calculated the structural parameters from this data. M.C. and W.F. provided access to high-performance computing resources and expertise on research software, methods and workflow. N.C. performed the 3D late stage liquid-liquid phase separation simulations for a series of different volume fractions. D.M.C. undertook the FDTD analysis for the Xray intra-scale structures and the computer simulated Cahn-Hilliard probe structures. D. M.C. also built the micro-optical setup used to measure the reflectance and the transmission of the beetle scales. S.B., A.B., A.W., S.P., R.D., O.O.M. and A.J.P. conducted the kinetic SAXS measurements on the synthetic system and A.J.R. helped analyse the early stage USAXS data for the synthetic CA system. S.B., S.R.P., R.M.D, A.W. and A.J.P measured the SESANS signals from the biological and synthetic samples. A.J.P. wrote the initial draft of the manuscript with input and revisions from all authors.

\section{Additional information}

Supplementary information accompanies this paper at https://doi.org/10.1038/s42004019-0202-8

Competing interests: The authors declare no competing interests.

Reprints and permission information is available online at http://npg.nature.com/ reprintsandpermissions/

Publisher's note: Springer Nature remains neutral with regard to jurisdictional claims in published maps and institutional affiliations.

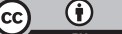

Open Access This article is licensed under a Creative Commons Attribution 4.0 International License, which permits use, sharing, adaptation, distribution and reproduction in any medium or format, as long as you give appropriate credit to the original author(s) and the source, provide a link to the Creative Commons license, and indicate if changes were made. The images or other third party material in this article are included in the article's Creative Commons license, unless indicated otherwise in a credit line to the material. If material is not included in the article's Creative Commons license and your intended use is not permitted by statutory regulation or exceeds the permitted use, you will need to obtain permission directly from the copyright holder. To view a copy of this license, visit http://creativecommons.org/ licenses/by/4.0/.

(C) The Author(s) 2019 